HEALTHY AORTA (WILD-TYPE)

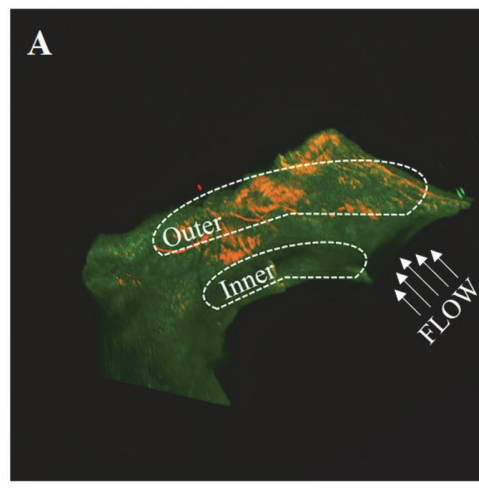

eNOS; VE-cadherin
PLAQUE (APOE--)

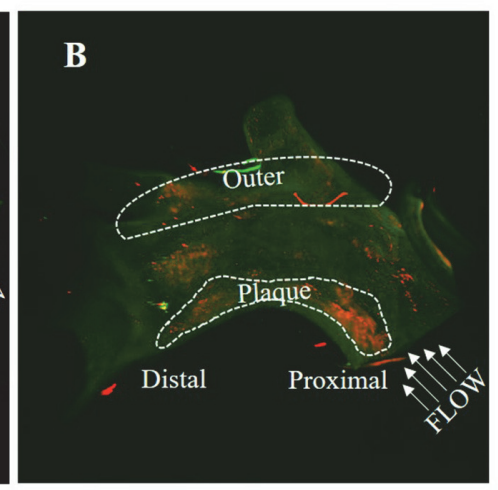

eNOS proximal vs dital

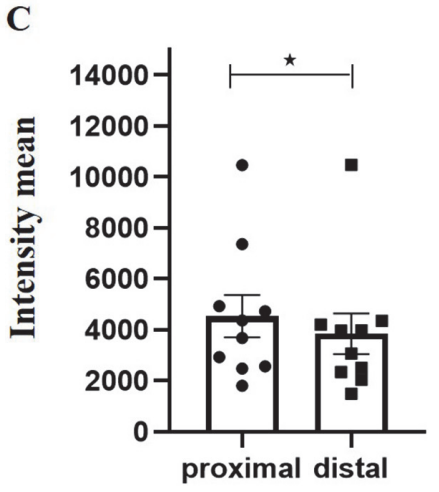

Abstract BS30 Figure 1

of the plaque compared to distal (downstream) suggesting a potential correlation with WSS (figure 1C).

Conflict of Interest Atherosclerosis

\section{BS31 VSMC CONTRIBUTION TO NEOINTIMAL LESIONS ARISES FROM THE CLONAL EXPANSION OF FEW PRIMED CELLS}

Matt Worssam. University of Cambridge, Cambridge, UK

\subsection{6/heartjnl-2021-BCS.229}

In healthy blood vessels, vascular smooth muscle cells (VSMCs) exist in a contractile, quiescent state but can switch phenotype to activate proliferation, migration and remodelling of the extracellular matrix. Phenotypically switched VSMCs contribute most cells within neointimal lesions, characteristic of atherosclerosis and in-stent restenosis, diseases that underlie heart attack and stroke. Using multicolour 'Confetti' VSMCspecific lineage tracing in animal models of vascular disease, we showed that the extensive VSMC contribution to these lesions results from the clonal expansion of few cells.

To understand how oligoclonal VSMC lesion contribution arises and to identify the signals activating VSMC proliferation in vivo, we used confocal microscopy to quantify VSMC clonal development over time in two models of vascular disease. We observed that the number and sizes of patches of clonally expanded VSMCs steadily increased, then plateaued post-injury. This suggests VSMC investment results from activation of a small number of VSMCs, rather than clonal competition following general VSMC activation. Selective VSMC activation in plaques was evidenced by the absence of plaques with high numbers of colours at any stage of plaque development.

In both models, VSMC activation was associated with vascular regions displaying elastic lamina alterations, medial acellularity and immune cell recruitment, implicating these as proliferation-triggering cues. However, not all VSMCs in these regions formed patches, suggesting that VSMCs must be primed to respond. In culture, few VSMCs gave rise to patches, suggesting cell-autonomous activation. This work supports the targeting of primed VSMCs in the healthy vessel as a therapeutic strategy against vascular lesion development.

Conflict of Interest None

\section{BS32 THE ROLE OF BRANCHED-CHAIN KETO ACIDS IN MEDIATING INSULIN RESISTANCE IN THE FAILING HEART}

Qutuba Karwi, Cory Wagg, Liyan Zhang, John Ussher, Gary Lopaschuk. University of Alberta, Edmonton, Canada

\subsection{6/heartjnl-2021-BCS.230}

Brief introduction: Perturbed branched-chain amino acids (BCAA) oxidation positively correlates with the severity of cardiac insulin resistance in heart failure. We previously demonstrated that cardiac-specific deletion of the BCAA oxidative enzyme mitochondrial branched-chain aminotransferase (BCATm) increases cardiac BCAA levels and decreases branched-chain keto acids (BCKA) levels, enhances insulinstimulated cardiac glucose oxidation rates. This increased cardiac insulin sensitivity is associated with an increase in the phosphorylation of protein kinase $\mathrm{B}$ (Akt) and activation of pyruvate dehydrogenase (PDH), the rate-limiting enzyme of glucose oxidation. However, whether it is the accumulation of BCAA or BCKA that is critical in mediating cardiac insulin resistance is unknown. How perturbed BCAA oxidation may mediate cardiac insulin resistance in heart failure is also unknown.

Explanation of basic Methods To address these questions, we first examined the effects of selectively enhancing cardiac BCKA levels on cardiac insulin-stimulated glucose oxidation. We perfused isolated working mice hearts (male and female C57BL/6N, $\mathrm{n}=8-10$ ) with high levels of BCKA ( $\alpha$-keto-isocaproate $80 \mu \mathrm{M}, \alpha$-keto- $\beta$-methylvalorate $100 \mu \mathrm{M}, \alpha$-keto-isovalerate $70 \mu \mathrm{M}$ ), levels that can be seen in diabetes and obesity.

Results High levels of BCKA completely blunted insulin-stimulated glucose oxidation rates and increased fatty acid oxidation rates. We also found that BCKA abolished insulin-stimulated mitochondrial Akt, an effect that was associated with PDH deactivation. We next determined the potential protective effect of reducing cardiac BCKA levels in the failing heart. We randomized WTCre $+/+$ and cardiac-specific BCATm- $/-$ mice (male, 25-30g, $\mathrm{n}=6-8$ ) to undergo either sham surgery or transverse aortic constriction surgery to induce heart failure. Five weeks post-surgery, there was a marked increase in insulin-stimulated glucose oxidation rates in the BCATm-/- failing hearts compared to the WTCre+/+ failing hearts, with no significant effect on glycolysis rates. Enhanced cardiac insulin 
sensitivity was associated with a significant decrease in fatty acid oxidation rates in the BCATm-/- failing hearts compared to the WTCre+/+ failing hearts. This decrease in fatty acid oxidation in the BCATm-/- failing hearts was associated with a significant decrease in myocardial oxygen consumption rates. As a result, cardiac efficiency (cardiac work/myocardial oxygen consumption) was significantly increased in the BCATm-/- failing hearts compared to the WTCre $+/+$ failing hearts.

Conclusions/implications We conclude that the accumulation of BCKA, and not BCAA, is a major contributor to cardiac insulin resistance via abrogating mitochondrial translocation of Akt. Targeting BCKA may represent a potential therapeutic approach to improve cardiac insulin-stimulated glucose oxidation in the setting of heart failure, obesity and diabetes. Conflict of Interest None

\section{BS33 THE RAF 'PARADOX BREAKER' INHIBITOR, PLX8394, ACTIVATES ERK1/2 IN ENDOTHELIAL CELLS AND PROMOTES CARDIAC REMODELLING}

${ }^{1}$ Susanna Cooper, ${ }^{2}$ Josh Cull, ${ }^{2}$ Angela Clerk, ${ }^{1}$ Daniel Meijles. ${ }^{1}$ St. George's University of London, London, UK; ${ }^{2}$ University of Reading, UK

\subsection{6/heartjnl-2021-BCS.231}

Introduction RAF kinases activate the ERK1/2 cascade, a key pathway involved in cardiac remodelling and cytoprotection. Since activating mutations in BRAF cause cancer, small molecule inhibitors of RAF have been developed. However, a paradoxical effect is observed with some inhibitors which activate rather than inhibit ERK1/2. Therefore, 'paradox breaker' inhibitors have been developed as new-generation cancer therapies void of this effect (e.g. PLX8394). Here, we determined the effects of PLX8394 on endothelial cell (EC) ERK1/ 2 signalling and the heart in vivo.

Methods Murine ECs were incubated with PLX8394 and effects on ERK1/2 activity determined by western blotting for the phosphorylated (i.e. activated) kinases. Effects on gene expression were determined by qPCR. The effects of PLX8394 on the heart in vivo were determined by infusing male wildtype C57Bl/6J mice (10-12wks, $\mathrm{n}=6 /$ group) with PLX8394 $(5 \mathrm{mg} / \mathrm{kg} / \mathrm{d}, 7 \mathrm{~d})$ using osmotic minipumps. Cardiac function/dimensions were assessed using echocardiography; effects on cardiac morphology were assessed by histological staining. mRNA expression was assessed by qPCR. Statistical tests used 1-way ANOVA with HolmSidak's post-test (in vitro studies) and unpaired t-tests (in vivo studies)

Results PLX8394 activated ERK1/2 in ECs in a time (7.4 \pm 2.3 -fold at $5 \mathrm{~min} ; \mathrm{p}=0.0365 ; \mathrm{n}=5)$ and concentration $(>1 \mathrm{uM} ; \mathrm{p}=0.0625 ; \mathrm{n}=3)$ dependent manner. This was associated with significant increases in expression of mRNAs encoding the immediate early gene Fos (6.1 \pm 2.6 -fold; $\mathrm{p}<0.0001 ; \mathrm{n}=4)$ and the vasoconstrictor peptide endothelin1 (Edn1) (2.7 \pm 0.9 -fold; $\mathrm{p}=0.0038 ; \mathrm{n}=4)$. In vivo, PLX8394 decreased cardiac output $(p=0.0092)$, predominantly through reduced stroke volume $(p=0.0103)$. Structurally, PLX8394 promoted cardiac hypertrophy, with increased diastolic left ventricular $(\mathrm{LV})$ posterior wall thickness $(\mathrm{p}=0.0425)$ and decreased LV internal diameter $(p=0.0463)$ at $7 \mathrm{~d}$. Cardiac hypertrophy resulted from increased cardiomyocyte cross-sectional area $(p=0.0002)$ despite no changes in Myh7, Nppa or Nppb mRNAs. Moreover, PLX8394-induced cardiac remodelling was not due to increased fibrosis, with no change in mRNA expression of collagens1-4 and using histological assessment.

Conclusion Despite being developed as a 'paradox breaker' for cancer, PLX8394 promoted ERK1/2 signalling in murine ECs and cardiac remodelling in vivo. These preliminary findings suggest that such inhibitors, currently in Phase 3 trials for RAF-mutant cancers, have potential to modulate cardiac function in patients.

Conflict of Interest N/A

\section{BS34 TELOMERE DAMAGE PROMOTES VASCULAR SMOOTH MUSCLE CELL SENESCENCE AND IMMUNE CELL RECRUITMENT AFTER VESSEL INJURY}

${ }^{1}$ Mandy Grootaert, 'Anna Uryga, ${ }^{1}$ Abel Garrido, 'Sebnem Oc, 'Kirsty Foote, 'Joel Chappell, ${ }^{1}$ Alison Finigan, ${ }^{2}$ Francesca Rossiello, ${ }^{2}$ Fabrizio d'Adda di Fagagna, 'Dimitra Aravani, ${ }^{1}$ Helle Jorgensen, ${ }^{1}$ Martin Bennett. ${ }^{1}$ University of Cambridge, Cambridge, UK; ${ }^{2}$ FIRC Institute of Molecular Oncology Foundation, IFOM Foundation, Milan

\subsection{6/heartjnl-2021-BCS.232}

Aim Vascular smooth muscle cells (VSMCs) accumulate in injury-induced neointimal lesions and atherosclerotic plaques in an oligoclonal fashion, yet plaque VSMCs show reduced proliferation and cell senescence. DNA damage leads to VSMC senescence and inflammation, and VSMC senescence promotes atherosclerosis; however, the exact mechanism by which VSMC senescence promotes lesion formation is not known. Here, we investigated telomere damage-induced VSMC senescence, the contribution of senescence-induced inflammation and the mechanisms involved, the consequences of VSMC senescence in vivo after injury, and whether it promotes clonality.

Methods Stress-induced premature senescence (SIPS) was induced by the chemotherapeutic doxorubicin (24h treatment $+21 \mathrm{~d}$ recovery). Lentiviruses were used to stably overexpress a dysfunctional TRF2 mutant protein (TRF2T188A) in human VSMCs (hVSMCs). SM22aTRF2T188A mice were generated that express human TRF2T188A in VSMCs only, and crossed with Myh11-CreERT2 Rosa26-Confetti multicolour reporter mice, to study cell senescence and clonality in vivo. Arterial injury was induced in these mice by ligation of the left common carotid artery for 28 days.

Results Both SIPS and TRF2188A-induced VSMC senescence were characterised by persistent telomere damage, and associated with formation of micronuclei, activation of cGAS-STING cytoplasmic DNA sensing, and induction of multiple proinflammatory cytokines. Silencing of cGAS in TRF2T188A hVSMCs partially inhibited NFkB-dependent cytokine expression. In vivo, VSMC-specific TRF2T188A expression in a multicolour clonal VSMC-tracking model demonstrated no change in VSMC clonal patches after injury, but increased neointima formation (figure 1), outward remodelling and cellular senescence. Moreover, neointimal lesions of VSMC-specific TRF2T188A mice were characterised by increased ICAM1 expression and increased abundance of CD45+, CD3 + and CD68 + cells when compared to littermate controls, indicating increased immune/inflammatory cell infiltration and/ or retention.

Conclusions Persistent telomere damage promotes VSMC senescence and inflammation, and exacerbates neointima formation after injury. Our data suggest that persistent telomere damage-induced VSMC senescence plays a major role in 